\title{
FAST VIDEO OBJECT DETECTION VIA MULTIPLE BACKGROUND MODELING
}

\author{
Kin-Yi Yam ${ }^{1,2}$, Wan-Chi Siu ${ }^{1}$,Ngai-Fong Law ${ }^{1}$,Chok-Ki Chan ${ }^{2}$ \\ ${ }^{1}$ Department of Electronic and Information Engineering \\ The Hong Kong Polytechnic University, Hong Kong \\ ${ }^{2}$ TeleEye Holding Limited (Hong Kong), Hong Kong
}

\begin{abstract}
In this paper, a robust background extraction and novel object detection are proposed, which comprise of filtering operations to detect non background objects in a monitoring scene. Conventionally, a statistical background model is extracted by using a training sequence without foreground objects and the background model parameters are being updated continuously to adapt changes in the scene. However, it is not possible to require a monitoring scene to be static. Furthermore, static objects in the scene could be adapted into the background. Problems arise when static objects start to move again. The convention method would produce false alarms in the detection process. In our proposed algorithm, two background models are constructed by using N-bins histogram method to indicate short term and long term changes of the monitoring scene. We then apply background subtractions to the current frame to obtain two error frames, which are combined for objects detection and classification. Extensive experimental work has been done, results of which show that the present approach provides a better solution compared with the conventional approach, including to resolve the problem of re-active objects.
\end{abstract}

\section{INTRODUCTION}

Objects detection and tracking are the main issues in most of the video surveillance system, such as traffic monitoring, objects counting, etc. A possible way to detect moving objects is to segment background by subtraction initially, especially when the system uses static cameras. In order to implement background subtraction to extract moving objects, the background model becomes a critical issue that affects the performance of object detection and tracking. Usually, a proper background model should have the ability to handle different problems in real applications. In recent researches, there are many methods to generate background model, such as using a single $[1,2]$ or mixture of Gaussian distributions for each pixel in the background model [3-6]. However, by using the above methods to generate a background model, they assumed that the background model was trained by a training sequence in which with no foreground objects appear and the computational time is expensive. In most of the scenarios, the appearance of foreground objects is unavoidable, especially in the initial state of the tracking system. Also, problems arise when static objects start to move again, which would produce false alarms in the detection process. For example, reference [6] gives a study on background generation with moving objects. It has the effect of ignoring the re-active objects since the background was not updated when objects occurred. However, it is lacking in strategy to classify the stopped objects in the scene.

This paper gives interesting results of our work on intelligent video monitoring, with an emphasis on background extraction and moving object detection. New ideas have been included in our monitoring procedure which includes background modeling, segmentation of moving object(s) and object tracking. The remaining parts of this paper are organized as follows. Section 2 describes the background generation algorithm. Section 3 gives our novel detection approach. Finally, a conclusion is given in section 4 .

\section{BACKGROUND MODELING}

\subsection{N-bin Histogram model}

Background subtraction is a major technique for the detection of moving and stationary objects in a surveillance system. The quality of it directly affects the segmentation result. The method used in this paper is to generate a statistical background from the histogram of each pixel in the frame. We create a background image map that contains frequency-intensity histogram that shows the number of occurrences of an intensity of each pixel in a period of time. In order to reduce the computational time for real time processing, each pixel contains only an n-bins histogram $\mathrm{H}(\mathrm{x}, \mathrm{y})$. Each bin, for example, contains a rang of 16 pixel intensity values (i.e. $\{[0: 16], \quad[17: 33] \ldots[139: 255]\}$. The histogram then contains 16 bins in this case). Thus we have,

$\mathrm{H}(\mathrm{x}, \mathrm{y})=\left\{\mathrm{bin}_{1}, \mathrm{bin}_{2}, \mathrm{bin}_{3} \ldots, \mathrm{bin}_{\mathrm{N}}\right\}$

where $\mathrm{N}$ is the number of bins in the histogram. The histogram is used to count the occurrence of intensity values for each pixel. Let us assume that $\mathrm{BIN}_{\mathrm{tn}}$ is the accumulated number of occurrences of the pixel at location $(\mathrm{x}, \mathrm{y})$ in the $\mathrm{n}^{\text {th }}$ bin at time t. We have

$$
B I N_{t n}=\sum_{t} b_{t n} \quad \text { where } \quad b_{t n}=\left\{\begin{array}{l}
1 \text { if } I_{t}(x, y) \in b_{\text {in }} \\
0 \text { if } I_{t}(x, y) \notin \text { bin }_{n}
\end{array}\right.
$$

and $\mathrm{I}_{\mathrm{t}}(\mathrm{x}, \mathrm{y})$ is the pixel value at location $(\mathrm{x}, \mathrm{y})$, at time $\mathrm{t}$.

According to the above equation, the most frequently occurring intensity interval $\left(\right.$ bin $\left._{\mathrm{b}}\right)$ in the histogram can be obtained. In our assumption, the most frequently occurring intensity bin should have the largest likelihood of being the background pixel intensity interval. We also make use of the current incoming pixel value (at this interval) as the current background pixel value in the model, i.e.

$$
\mathrm{B}_{\mathrm{t}}(\mathrm{x}, \mathrm{y})=\mathrm{I}_{\mathrm{t}}(\mathrm{x}, \mathrm{y}) \in \text { bin }_{\mathrm{b}}
$$

$\mathrm{B}_{\mathrm{t}}(\mathrm{x}, \mathrm{y})$ is the background pixel value at location ( $\mathrm{x}$, y) at time $t$, and $\operatorname{bin}_{b}$ is the background bin. Fig 1 illustrates this background generation process.

In fig.1, each pixel contains a histogram on seeking for the suitable background pixel values in the 
background frame. For example, the histogram contains 256 intensity values. It is quantized into 16 levels, each level contain 16 intensity values. The most frequent occurring bin becomes the background bin. In fig. 2 the most active intensity interval indicated is $20-40$, therefore this interval is chosen as the background interval, and the value of that pixel is the latest value at that interval such as 35 for frame 439. In the case for those intensity values that do not fall into the most active intensity internal, such as the blue circles in the graph, it indicate that there is a moving object containing this pixel. The background pixel value of this location then remains the same during this active intensity interval. Subsequently the background frame can be constructed. Due to this arrangement of background modeling, some forms of the "moving" background, such as the escalators in some shopping mall, can be visually seen as the moving backgrounds in this model. This method also has the advantage that the background pixel values are close to the current values, and false detection can be minimized.

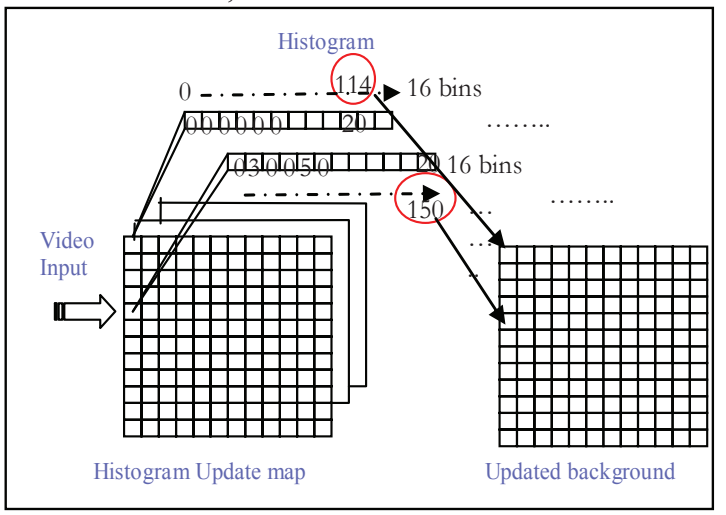

Figure 1. Example showing the algorithm of background generation.

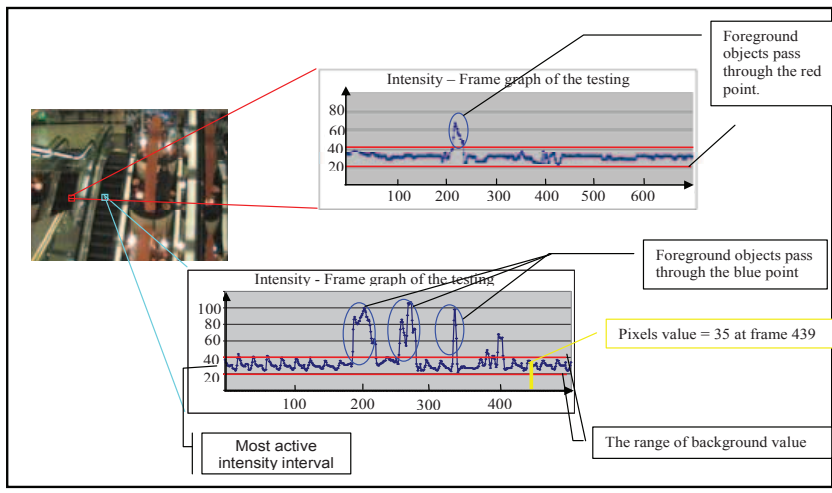

Figure 2. Gaphic showing a possible pixel values distribution pixels at (a) red and (b) blue rectangles on the frame (left) in the time domain 2.2. Background model update

In real life application, the background would not be the same all the time. The scene changes continuously, such as the illumination change at outdoor environment and the movement of the background. Objects that come into the scene may stay for a long time. In order to adapt these kinds of issues, background update is needed. In our background modeling method, the background frame is started to update immediately when it has been created. Each bin will have an upper bound record of occurrences of pixel values. If any acuminated value of the histogram reaches a pre-determined value, the histogram is reset. All bins are then reset to zero except the one that belongs to background bin at time t-1. The background will be set to half of the pre-determined value. The reason is that if the bin is considered as a background bin, it is assumed to be a background bin also at time $t+1$. This arrangement makes the background bin remain its function after the reset

Recall the equation (2) and note that $\mathrm{BIN}_{\mathrm{tn}}<\mathrm{P}$, where $\mathrm{P}$ is the pre-determined value, then,



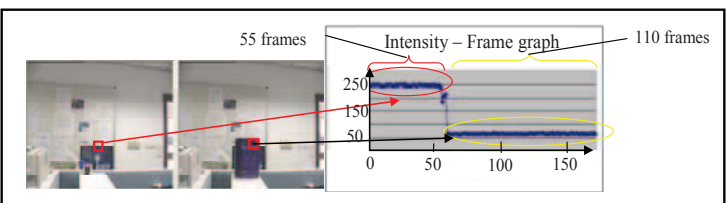

Figure 3. An example of adaptive background: (a) a scene in the DS Laboratory at time $\mathrm{t} 1$ (frame 1) and at time $\mathrm{t} 2$ (frame 112), and (b) Graph of the intensities of the marked pixels against the frame numbers.

According to the above arrangement, the background bin will change according to its bin's accumulated value $\left(\mathrm{BIN}_{\mathrm{tn}}\right)$. It means that the most active intensity interval can be modified. The criterion is that if the accumulated value of any of its non-background bins is bigger then the current accumulated value of the background bin, the bin will become a new background bin

Let $\operatorname{bin}_{b}$ be the background bin with accumulative value $\mathrm{BIN}_{\mathrm{tb}}$, and then it can be written as:

$$
\operatorname{bin}_{n}=\left\{\begin{array}{l}
\operatorname{bin}_{b} \text { if }\left(B_{I N} \in H(x, y) \mid n \neq b\right)>B I N_{t b} \\
\operatorname{bin}_{n} \text { if }\left(\operatorname{BIN}_{t n} \in H(x, y) \mid n \neq b\right) \leq B I N_{t b}
\end{array}\right.
$$

The procedure of background modeling:

- Start the video sequence (either offline or real-time video).

- Prepare a histogram (pixel intensity against frame number) for each pixel.

- Update continuously the histogram for every frame

- Find the most frequently occurring bin in the histogram of each pixel in each update. The current intensity values within the background bin become the background pixels.

\subsection{Short term and long term background models}

Our detection method based on background modeling is to make use of two background models. One of them represents short-term background and the other is for long term background. The differences between the two backgrounds are the pre-determinate values of the bins. The short-term background contains a smaller value, which means the background bin is easily to be replaced by another bin in the histogram. On the other hand, the long-term background contains a larger value, so the background is not easily to be updated. Once an object is detected, the detected area in the long-term background model may not be updated. Therefore the long-term background usually does not contain temporally adapted foreground objects.

Our method using short-term and long-term backgrounds for background subtraction, not only can classify active and static objects in the scene, but also classify "re-active" regions in the scene in order to 
reduce the false detection. After background subtraction, two different error frames are produced $\mathrm{E}_{1 \mathrm{t}}(\mathrm{x}, \mathrm{y})$ and $\mathrm{E}_{2 \mathrm{t}}(\mathrm{x}, \mathrm{y})$ in time $\mathrm{t}$. These two error frames contain different characteristics. By combining the patterns of the two error frames, two new maps are generated at time $t$. We call them final error map, $\mathrm{E}_{\mathrm{ft}}(\mathrm{x}, \mathrm{y})$ and classified map $E_{c t}(x, y)$. Final error map $E_{f t}(x, y)$ acts as a logic function of $E_{1 t}(x, y)$ and $E_{2 t}(x, y)$.

Classified map $\mathrm{E}_{\mathrm{ct}}(\mathrm{x}, \mathrm{y})$ contains 4 different representations for each pixel. They are background, active, static and "re-active" pixels. The corresponding numbers are $0,1,2$ and 3 . For those error fames, each of them is a binary pattern frame with the pixel value either equals 1 or 0 at time t.

$$
E_{t}(x, y)= \begin{cases}0 & \text { if }\left|I_{t}(x, y)-B_{t}(x, y)\right|<\text { threshold } \\ 1 & \text { otherwise }\end{cases}
$$

where $\mathrm{E}_{\mathrm{t}}(\mathrm{x}, \mathrm{y})$ is the error frame, and the threshold is equal to 20. The threshold was deduced from experimental results.

Both maps contain important information on pixels states, which enhance the ability of the approach to classify pixels for object detection. The following table shows the patterns and the corresponding physical meanings.

TABLE I. THE COMBINATION PATTERN AND THE PHYSICAL MEANING OF TWO BASIC ERROR FRAMES. (*THIS TABLE SHOWS THE POSSIBLE STATUS OF A PIXEL IN THE FRAME ONLY)

\begin{tabular}{|c|c|c|c|l|}
\hline $\begin{array}{c}\text { Error } \\
\text { Frame1 } \\
\mid \mathbf{I}(\mathbf{x}, \mathbf{y})- \\
\mathbf{B}_{\text {short-term(x,y) }} \mid\end{array}$ & $\begin{array}{c}\text { Error } \\
\text { Frame2 } \\
\mid \mathbf{I}(\mathbf{x}, \mathbf{y})-\mathbf{B}_{\text {long- }} \\
\text { term(x,y) } \mid\end{array}$ & $\begin{array}{c}\text { Final } \\
\text { Error } \\
\mathbf{M a p} \\
\left(\mathbf{E}_{\mathbf{f t}}(\mathbf{x}, \mathbf{y})\right)\end{array}$ & $\begin{array}{c}\text { Classified } \\
\mathbf{M a p} \\
\left(\mathbf{E}_{\mathrm{ct}}(\mathbf{x}, \mathbf{y})\right)\end{array}$ & $\begin{array}{c}\text { Physical } \\
\text { meaning }\end{array}$ \\
\hline 0 & 0 & 0 & 0 & $\begin{array}{l}\text { Background } \\
\text { pixel }\end{array}$ \\
\hline 1 & 1 & 1 & 1 & Active pixel \\
\hline 0 & 1 & 1 & 2 & Static pixel \\
\hline 1 & 0 & 0 & 3 & Re-active pixel \\
\hline
\end{tabular}

The column $\mathrm{E}_{\mathrm{ct}}(\mathrm{x}, \mathrm{y})$ shows that the classification of the pixel states. The corresponding "physical meanings" are shown at the last column.

After the above arrangement, the final error map will be used for objects detection. The detected result as shown indicates that the detected object is either moving object or a static object. The advantage of this arrangement is that if the static object starts moving again, the "hole" left behind the object will not be detected.

In addition, different applications will have different arrangements of the final error map. In the above cases, we would like to detect both active and static objects, which means only the pixels defined as 1 and 2 in the classified map will be detected. On the other hand, if we just want to detect re-active object, then only the pixels defined as 3 in the classified map will be detected. This approach is suitable for different applications, such as stolen object detection and "left" person detection, such that many false alarms can be eliminated.

\section{OBJECT'S DETECTION AND SEGMENTATION}

The above background generation is used to segment foreground objects from a video scene. By subtracting the constructed background model from the current frame, the foreground objects will appear in the scene and can be detected, using equation (6).
In the previous section, we have shown the way to create the final error map. However usually there contains a lot of noise due to the performance of the camera and some unwanted small movement in the scene. All these problems affect the segmentation result.

In order to resolve this problem, usually in many others paper, the morphological operation is applied to the error frame. The erosion and dilation operations can eliminate noises and small movement in the frame. However, the computational time would increase dramatically. In our object detection method, simple filtering and detection process are used together. Our object detection algorithm makes use of the basis of morphological operation, and it is unlike the conventional connected component method to detect segmented object that need a clear error pattern frame and have to work through every foreground pixels. Our detection algorithm is robust to noisy environment.

\subsection{Robust object's detection}

At the start of searching, a detector is built in order to help for searching objects in the error frame. The error frame used here is the Final error frame $\mathrm{E}_{\mathrm{ft}}(\mathrm{x}, \mathrm{y})$ for which we have mentioned in the previous section. The detector, for example, is a $3 \times 3$ matrix with the value of one except the center value is minus 8 . This detector starts searching the error frame from top to bottom and from left to right. The error pattern is considered as an object, once the detector can fully cover the error pattern, which means the sum of the matrix is zero. A mathematic expression can be written as:

$$
\begin{aligned}
& \vec{C}_{d}=\left(\begin{array}{ccc}
1 & 1 & 1 \\
1 & -8 & 1 \\
1 & 1 & 1
\end{array}\right) \otimes\left(\begin{array}{lll}
e_{00} & e_{01} & e_{02} \\
e_{10} & e_{11} & e_{12} \\
e_{20} & e_{21} & e_{22}
\end{array}\right)=\left(\begin{array}{ccc}
c_{00} & c_{01} & c_{02} \\
c_{10} & c_{11} & c_{12} \\
c_{20} & c_{21} & c_{22}
\end{array}\right) \\
& \sum_{i=0}^{2} \sum_{j=0}^{2} c_{i j}=\left\{\begin{array}{cc}
0 & \text { if } \forall e_{i, j}=1 \\
0<\text { any values } \leq 8 & \text { otherwise }
\end{array}\right.
\end{aligned}
$$

where $\otimes$ Indicates element-by-element multiplication.

Once the detector has found an error pattern, the location at the center of the block matrix becomes the starting point of next operation called boundary-seeking operation. This process consists of two parts. The first part is to find the initial boundary of the object that may not cover the whole object as shown in Fig 4(c). Further seeking of the object boundary is the second part of the whole boundary-seeking operation in Fig 4(d). The advantages of this method are time saving and the noise in the frame would also be substantially reduced.

The initial boundary of the object is found from the center of the "detector" matrix, $\mathrm{c}_{11}(\mathrm{x}, \mathrm{y})$, where $\mathrm{x}$ and $\mathrm{y}$ indicate the corresponding location in the frame. Firstly, the initial left and right boundaries of the object are found by expanding horizontally the searching position from $\mathrm{c}_{11}(\mathrm{x}, \mathrm{y})$; similarly, the top and bottom boundaries are found by expanding vertically the searching position from $\mathrm{c}_{11}(\mathrm{x}, \mathrm{y})$. Therefore we can obtain the four corners: the top $\left(X_{T}, Y_{T}\right)$, bottom $\left(X_{B}, Y_{B}\right)$, left $\left(X_{L}, Y_{L}\right)$, and right edge $\left(X_{R}, Y_{R}\right)$ as shown in Fig. 4(b). Then a rectangle can be drawn. In order to get the whole object, the second step of searching the boundary is introduced. The search will start from the four edges, if any error pattern is found nearby the edges, the old locations at the top, bottom, left and right will be replaced by the new locations, $\left(X^{\prime}\right.$, $\left.\mathrm{Y}_{\mathrm{T}}{ }_{\mathrm{T}}\right),\left(\mathrm{X}_{\mathrm{B}}{ }_{\mathrm{B}}, \mathrm{Y}_{\mathrm{B}}{ }_{\mathrm{B}}\right),\left(\mathrm{X}_{\mathrm{L}}^{\prime}, \mathrm{Y}_{\mathrm{L}}{ }_{\mathrm{L}}\right)$, and $\left(\mathrm{X}_{\mathrm{R}}{ }_{\mathrm{R}}, \mathrm{Y}_{\mathrm{R}}{ }_{\mathrm{R}}\right)$. Hence, the 
new object boundary can be found. This is an iterate process, until none of the error pixel is detected from the four edges. As a result the whole object could be found. The graphic description shows in Fig 4.

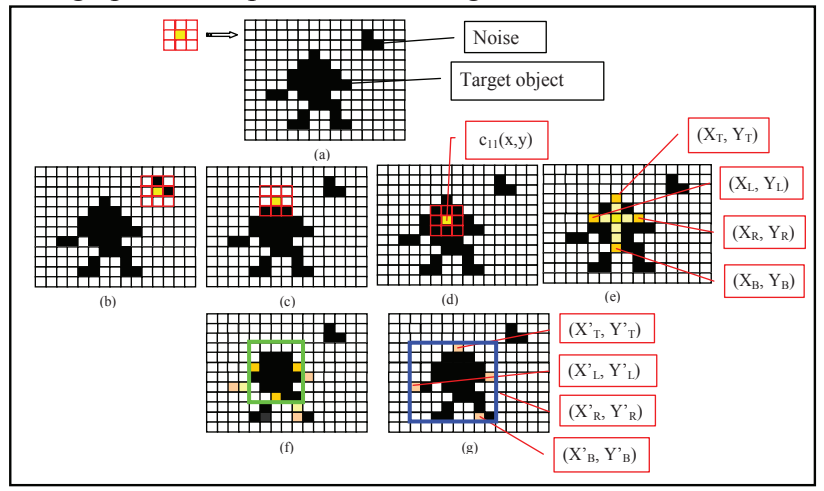

Figure 4. Object Extraction: (a) A frame containing noise and the target object. (b) The detector matrix searching from left to right, top to bottom, and reaching the noise pattern. (c) Further search and reaching part of the object. (d) The target object occupied fullfy the target object. (The location with yellow color is $\mathrm{c}_{11}(\mathrm{x}, \mathrm{y})$ ). (e) Four corners. (f) The green boundary formed temporally by the four corner locations. (g) Further search from four green edge found in (f)

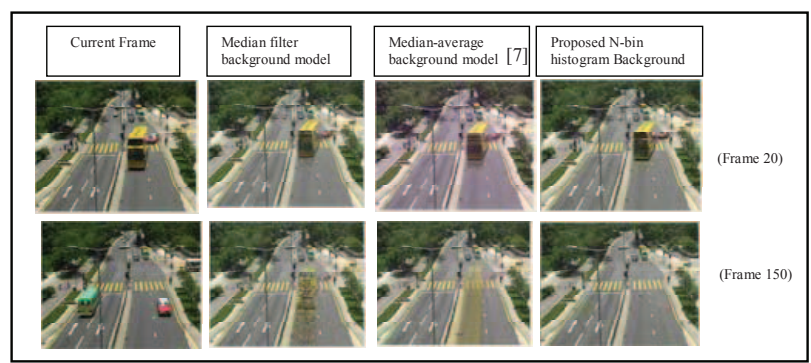

Figure 5. Comparsion results of the conventional, reference [7] and proposed mothods.

\section{EXPERIMENTAL RESULT}

Fig. 5 shows the number of frames required to create a clear adaptive background. We can see that our approach can create clear background at a speed faster than using other approaches. In our test, other background models [7] required up to 800 frames to create a clear background. At frame 150, our approach provided a clear background already. It is due to the pixel intensities of the moving objects are not used at an early stage. Therefore the pixels value outside the background bin will not be considered as background pixels values. On contrary, the median and media-average background models still contain some blurred areas on the road, due to the moving object - the yellow bus in this case, appeared in the scene initially.

Fig.6 shows different results between the conventional [8] and the proposed N-bins histogram adaptive background models. The testing sequence contains an initial moving vehicle on the road. At the 470th frame the vehicle stopped on the road and lasted for 230 frames. After then the vehicle started to move again until it left the scene. The conventional N-bins histogram adaptive background can detect and track the moving vehicle. When it stopped for a while, it was adapted into the background. Thus, the stopped vehicle will no longer be detected. When it moves again, false detection occurred as shown in Fig. 6(b). However, the proposed algorithm can classify the static vehicle and the re-active vehicle as shown in Fig. 6(c) to 6(e), which is an effective means to reduce the false detection.

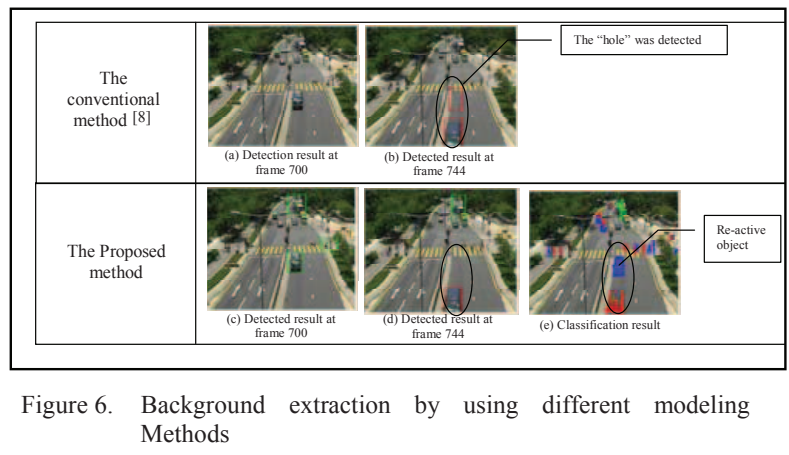

\section{CONCLUSION}

This paper makes use of the technique of background subtraction to segment out moving and stationary objects. Adaptive background is constructed to suit for different applications. A novel object detection method that combines filtering and searching methods, in order to reduce the computational time is proposed. The approach is robust to noisy environment and has been found suitable for various kinds of real-time applications.

\section{REFERENCES}

[1] C. Xun, J. Long, H. Xing-wei, and M. Xiang-xu, "A New Region Gaussian Background Model for Video Surveillance," Proceedings, pp. 123-127, Vol 6, fourth international conference on Natural Computation, 2008, Jinan.

[2] G. Bailo, M. Bariani, P. Ijas, and M. Raggio, "Background estimation with Gaussian distribution for image segmentation, a fast approach," Proceeding, pp.2-5, IEEE International Workshop on Measurement Systems for Homeland Security, Contraband Detection and Personal Safety Workshop, March 2005

[3] L. Dar-Shyang, "Effective Gaussian mixture learning for video background subtraction," pp.827-832, Vol.27, IEEE Transactions on Pattern Analysis and Machine Intelligence, May 2005.

[4] Shimada, D. Arita, and R. Taniguchi, "Dynamic Control of Adaptive Mixture-of-Gaussians Background Model," Proceedings, pp. 5, IEEE International Conference on Video and Signal Based Surveillance, Nov 2006, Sydney, Australia.

[5] Dorin Comaniciu, Peter Meer. "Kernel-Based Object Tracking". Pp.564-577 Vol. 25, IEEE Transactions on pattern analysis and Machine Intelligence, May 2003.

[6] H. Hu and Z. Li, Z, Qu and D.Wang, "Vision-based moving objects setection with background modeling" p.p.436-439, Vol.2, IEEE International Conference on Measuring Technology and Mechatronics Automation, April, 2009.

[7] S. Indupalli, M.A.Ali, B.Boufama. "A Novel ClusteringBased Method for Adaptive Background Segmentation", p.p.37-37, The $3^{\text {rd }}$ Canadian Conference on Computer and Robot Vision, Jane, 2006.

[8] P. Wei, W. Kun, C. Zhi, and Y. Zhi-Sheng, "A Background Reconstruction Method Based on Doublebackground," pp. 502-507, Int. Conference on Image \& Graphics, Aug 2007, Sichuan, China. 